\title{
Drug Treatment of Benign Prostatic Hyperplasia and Hospital Admission for BPH-Related Surgery
}

\author{
P.C. Souverein ${ }^{\mathrm{a}, *}$, J.A. Erkens ${ }^{\mathrm{b}}$, J.J.M.C.H. de la Rosette ${ }^{\mathrm{c}}$, H.G.M. Leufkens ${ }^{\mathrm{a}}$, \\ R.M.C. Herings ${ }^{a, b}$ \\ ${ }^{a}$ Department of Pharmacoepidemiology and Pharmacotherapy, Utrecht Institute for Pharmaceutical Sciences, \\ P.O. Box 80082, 3508 TB Utrecht, The Netherlands \\ ${ }^{\mathrm{b}}$ PHARMO Institute for Drug Outcomes Research, Utrecht, The Netherlands \\ ${ }^{\mathrm{c}}$ Department of Urology, Academic Medical Center, Amsterdam, The Netherlands \\ Accepted 13 February 2003
}

\begin{abstract}
Objective: To investigate whether there is a difference in the risk of progressing to BPH-related prostatic surgery between patients using alpha-blockers and patients using the 5-alpha-reductase inhibitors (5-ARIs).

Methods: A population-based cohort study was conducted, using data from the PHARMO Record Linkage System. We identified 5671 patients ( $\geq 50$ years old, no history of using both alpha-blockers and 5-ARIs, more than one year of database history prior to the first date of BPH drug-dispensing), who filled at least one prescription for either alpha-blockers (alfuzosin, tamsulosin, terazosin) or 5-ARIs (finasteride). The incidence of BPH-related surgery was compared between patients treated with alpha-blockers and patients treated with 5-ARIs.

Results: The cumulative incidence of BPH-related prostatic surgery was $15.2 \%$ and mainly involved transurethral resection of the prostate (TURP) (13.4\%). Patients using alpha-blockers had a significantly increased risk of BPHrelated prostatic surgery compared to patients using 5-ARIs, which remained after adjusting for age, calendar time, type of prescriber and chronic disease score (adjusted HR: 1.52, 95\% CI: 1.24-1.88). The difference between alphablockers and 5-ARIs was sustained after stratification of time period $(<1995, \geq 1995)$ and exclusion of patients with prostatic surgery within one month of treatment initiation.

Conclusions: It is concluded that alpha-blocker treated patients had a higher risk of BPH-related surgery compared to 5-ARI treated patients. Additional research on the long-term outcomes and risk factors for the natural progression of BPH is necessary to identify the optimal medical treatment for BPH patients according to their baseline characteristics.
\end{abstract}

(C) 2003 Elsevier Science B.V. All rights reserved.

Keywords: Population-based study; Cohort study; Alpha-blockers; 5-alpha-reductase inhibitors; Benign prostatic hyperplasia; BPH-related surgery; Transurethral resection of the prostate

\section{Introduction}

Benign prostatic hyperplasia (BPH) is a common histological condition that can be detected in $90 \%$ of men aged over 80 years [1]. Patients with symptomatic $\mathrm{BPH}$ suffer from a large set of symptomatic scenarios of

* Corresponding author. Tel. +31-30-253-6976/7324;

Fax: +31-30-253-9166.

E-mail address: p.c.souverein@pharm.uu.nl (P.C. Souverein). lower urinary tract symptoms (LUTS), benign prostatic enlargement and bladder outlet obstruction [2]. Treatment of symptomatic BPH can be categorised in three distinct modalities: watchful waiting (no active treatment), pharmacotherapy and surgical intervention. Surgical procedures, including transurethral resection of the prostate (TURP), are the gold standard in BPH treatment, but medical therapy has gained increasing popularity since the early 1990s. This change is reflected by a time-trend graph of the prevalence of BPH drug use 


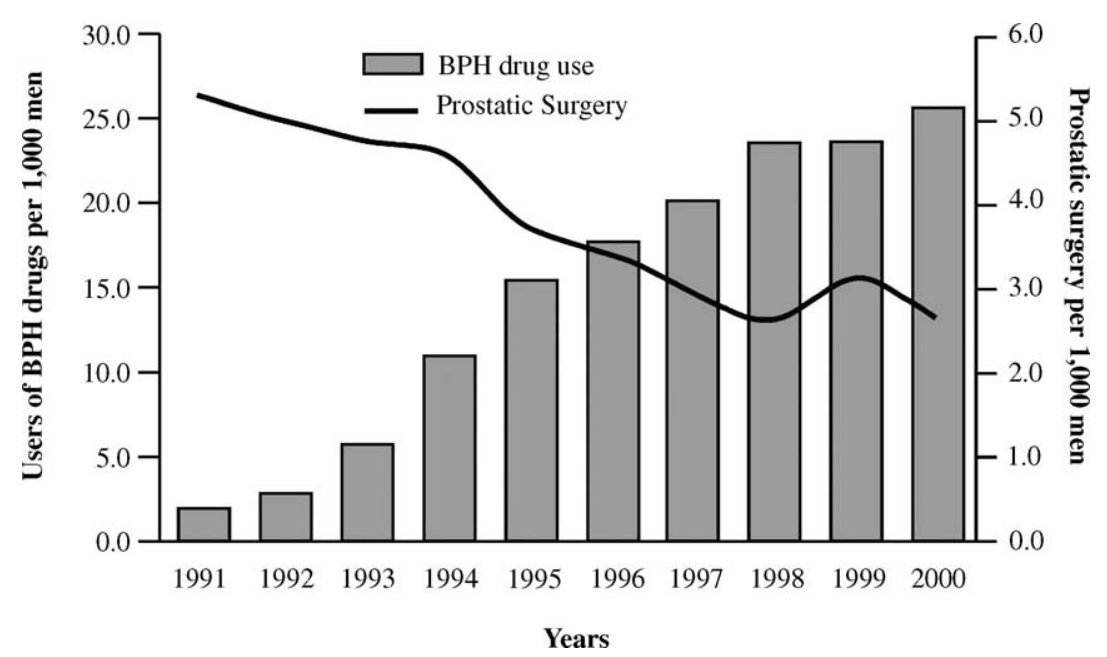

Fig. 1. BPH drug use and prostatic surgery in the PHARMO area 1991-2000 (source: PHARMO Institute for Drug Outcomes Research).

and prostatic surgery in the period 1991-2000 among men aged 50 years and older (Fig. 1). The prevalence of BPH drug use increased, while the prevalence of prostatic surgery decreased statistically significantly in the same period (source: PHARMO Institute for Drug Outcomes Research).

Clinical trials have shown that 5-alpha-reductase inhibitors (5-ARIs) and alpha-blockers are effective in reducing LUTS suggestive of BPH and improving urinary flow [3]. Alpha-blockers reduce smooth muscle tone in the bladder neck and the prostate, while 5-ARIs inhibit the conversion of dihydotestosteron to testosterone and, as a result, shrink the prostate. Alphablockers have a rapid effect on LUTS (in the order of weeks), while benefit of 5-ARIs can be expected after about six months. Open-label clinical trial extensions have shown that the efficacy of drug treatment is maintained after three years to five years [4-7].

However, the question remains whether drug treatment is capable of reducing the need for surgical intervention or delaying the inevitable. Recent data from the Medical Therapy of Prostatic Symptoms Study (MTOPS), a randomised, placebo-controlled clinical trial, demonstrated a greater reduction in the risk of acute urinary retention (AUR) and invasive surgery for finasteride compared with the alphablocker doxazosin [8]. Because clinical trial populations are known to be different from patients in daily clinical practice, observational studies are a valuable complement to clinical studies in assessing benefit (and risks) of marketed drugs [9,10]. Therefore, we assessed whether there was a difference in the risk of progressing to BPH-related prostatic surgery between patients using alpha-blockers and 5-ARIs in a population-based study.

\section{Methods}

\subsection{Data setting}

Data for this study were obtained from the PHARMO Record Linkage System (RLS) in The Netherlands. The PHARMO RLS includes the drug-dispensing records from community pharmacies and hospital discharge records of all 950,000 community-dwelling inhabitants of 25 medium-sized cities in the Netherlands. For all residents, the drug-dispensing histories are linked to the hospital discharge records of the same patient, using a probabilistic algorithm, based on characteristics such as date of birth, gender, and a code for the general practitioner. Validation shows that these registries are linked with a sensitivity and specificity exceeding $95 \%$ that is comparable to record linkage systems based on unique personal identifiers $[11,12]$. The computerised drug-dispensing histories contain data concerning the dispensed drug, type of prescriber, dispensing date, dispensed amount, prescribed dose regimens, and the legend duration (prescription length). The hospital records include detailed information concerning the primary and secondary diagnoses, procedures, and dates of hospital admission and discharge. All diagnoses are coded according to the ICD-9-CM (International Classification of Diseases, 9th Revision, Clinical Modification). Drug names are coded according to the Anatomical Therapeutic Chemical (ATC) Classification.

\subsection{Cohort definition}

The cohort comprised men aged 50 years or older who had filled at least one prescription for a drug indicated for the treatment of BPH in the Netherlands between 1991 and 2000. BPH drugs included were the selective alpha-blockers alfuzosin, tamsulosin, and terazosin, as well as the 5-alpha-reductase inhibitor finasteride. Patients using the alpha-blockers doxazosin and prazosin were excluded from the cohort, as these are non-specific BPH drugs having hypertension as their main indication. To ensure that the first prescription represented incident BPH drug use, only men who had been registered in the PHARMO RLS for at least one year before the first prescription of a BPH drug were eligible for inclusion in the cohort. Patients who switched from one BPH treatment to another or who were dispensed two active BPH treatments concurrently were excluded from the analysis. Furthermore, patients with a history of BPH-related surgeries or hospitalisations prior to starting 
$\mathrm{BPH}$ drugs were not considered eligible for the study cohort (definitions listed in Appendix A).

\subsection{Definition of the outcomes}

BPH-related outcomes were defined as procedures for incision of prostate (procedure-code 5-600), transurethral resection of the prostate (TURP) (procedure-code 5-601), suprapubic or retropubic prostatectomy (procedure-codes 5-602 and 5-603), prostatectomy (5-604), or other prostatic surgery (5-605) (see Appendix A).

\subsection{Data analysis}

The Cox proportional hazards model was used to assess the relation between BPH drugs and hospital admissions for surgical treatment of BPH among patients using exclusively an alphablocker and patients exclusively using finasteride. Hazard ratios (HR) were used to estimate relative risks (RR) and 95\% confidence intervals (CI). In the Cox regression analysis, finasteride was taken as reference category. The study patients entered in the cohort at the date of the first BPH drug prescription and were followed until a $\mathrm{BPH}$ outcome or censored. Patients were censored at the end of their registration period or at the end of the study period (31 December 2000). Hazard ratios were adjusted for potential confounders, including age, calendar year, type of prescriber and chronic disease score. The chronic disease score (CDS) was used as a proxy for general health and was calculated by assigning scores (0-5) to classes of drugs according to the severity of the disease for which they were prescribed during a period of one year [13]. Because finasteride has been shown to be effective after six months of treatment and the efficacy of alpha-blockers can be assessed within about four weeks [14], the follow-up of patients was stratified in different times periods: four weeks, 13 weeks and to the end of the observation period. To assess whether results were similar according to calendar time, we stratified the periods into before and after 1995 .

\section{Results}

There were 13,851 men with at least one prescription of one of the four BPH drugs between 1991 and 2000 in the PHARMO area $(n=950,000)$, of whom 5671 were considered eligible for inclusion in the study. The characteristics of the study population are shown in Table 1 . The mean follow-up time per patient was 1025 days (2.8 years). The mean age of the patients was 67 years, $76.5 \%$ of whom were older than 60 years. Most patients were initially treated with an alpha-blocker (85.4\%). Drug therapy was usually initiated by general practitioners $(51.5 \%)$
Table 1

Patient characteristics of the cohort of BPH drug users $(n=5671)$

\begin{tabular}{|c|c|}
\hline Characteristic & Number $(\%)$ \\
\hline \multicolumn{2}{|l|}{ Age at baseline (years) } \\
\hline $50-54$ & $597(10.5)$ \\
\hline $55-59$ & $745(13.1)$ \\
\hline $60-64$ & $876(15.5)$ \\
\hline $65-69$ & $1088(19.2)$ \\
\hline $70-74$ & $961(17.0)$ \\
\hline $75-79$ & $775(13.7)$ \\
\hline $80-84$ & $406(7.2)$ \\
\hline$\geq 85$ & $223(3.9)$ \\
\hline \multicolumn{2}{|l|}{ Year at baseline } \\
\hline 1992 & $48(0.9)$ \\
\hline 1993 & $234(4.1)$ \\
\hline 1994 & $545(9.6)$ \\
\hline 1995 & $624(11.0)$ \\
\hline 1996 & 617 (13.7) \\
\hline 1997 & 774 (13.4) \\
\hline 1998 & 1035 (18.3) \\
\hline 1999 & $867(15.3)$ \\
\hline 2000 & $927(16.4)$ \\
\hline \multicolumn{2}{|l|}{ BPH drug during follow-up } \\
\hline 5-ARI only & $831(14.7)$ \\
\hline Alpha-blocker only & $4840(85.4)$ \\
\hline \multicolumn{2}{|l|}{ Prescriber at baseline } \\
\hline General practitioner & $2918(51.5)$ \\
\hline Urologist & $2424(42.7)$ \\
\hline Other medical specialist & $329(5.8)$ \\
\hline \multicolumn{2}{|l|}{ BPH-related surgeries (total follow-up) } \\
\hline Incision of prostate & $1(0.1)$ \\
\hline Transurethral resection of the prostate & $759(13.4)$ \\
\hline Suprapubic/retropubic of prostatectomy & $42(0.7)$ \\
\hline Prostatectomy & $52(0.9)$ \\
\hline Other & $5(0.1)$ \\
\hline
\end{tabular}

5-ARI, 5-alpha-reductase inhibitor.

and urologists $(42.7 \%)$. During the follow-up period $15.2 \%$ of the patients underwent BPH-related surgeries, predominantly TURPs $(n=759,13.4 \%)$.

The Kaplan-Meier curve (Fig. 2) shows the proportion of patients remaining free of BPH-related prostatic surgery over time for 5-ARI treated patients compared to alpha-blocker treated patients. Patients using alphablockers had a significantly increased risk of BPHrelated prostatic surgery compared to patients using a 5-ARI (adjusted HR: 1.52, 95\% CI: 1.24-1.87) (Table 2). The hazard ratio relates to a number needed

\section{Table 2}

Survival from first BPH drug treatment to BPH-related surgery

\begin{tabular}{lccc}
\hline BPH drug use & Number of patients & Number of events $(\%)$ & Crude HR (95\% CI) \\
\hline 5-ARI & 831 & $109(13.1)$ & 1.00 (reference) \\
Alpha-blockers & 4840 & $750(15.5)$ & $1.00($ reference) \\
\hline
\end{tabular}

HR, hazard ratio; CI, confidence interval; 5-ARI, 5-alpha-reductase inhibitor.

${ }^{\text {a }}$ Adjusted hazard ratio for age, calendar year, type of prescriber and chronic disease score. 


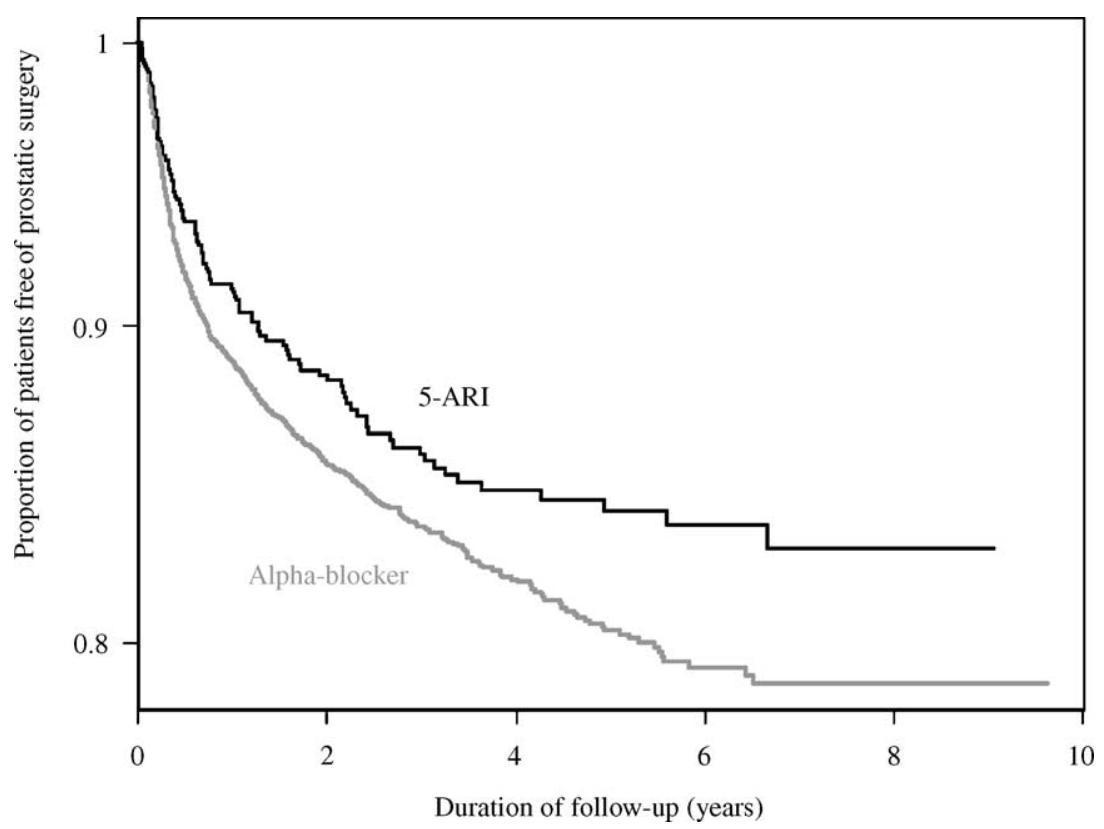

Fig. 2. Kaplan-Meier survival plot for patients using 5-alpha-reductase inhibitors and patients using alpha-blockers.

to harm $(\mathrm{NNH})$ of 14 (95\% CI: 9-30) after four years, meaning that one additional case of prostatic surgery occurs for every 14 patients treated with alpha-blockers for four years relative to 5-ARI treated patients. Similar results were obtained when excluding patients with a diagnosis of prostate cancer or patients having cryo surgery (adjusted HR: 1.67, 95\% CI: 1.34-2.08).

In Table 3, patients were stratified according to duration of treatment. For patients using alpha-blockers, a higher risk of $\mathrm{BPH}-$ related prostatic surgery compared with 5-ARI treated patients was observed in both duration periods of $<365$ days and $\geq 365$ days that increased, although not significantly, with duration: adjusted HR 1.17 (95\% CI: 0.93-1.49) and adjusted HR 1.53 (95\% CI: 0.95-2.47), respectively. When restricting the analysis to patients who were dispensed drugs later than 1995 and subsequently earlier than or in 1995, similar results showing a higher risk of patients treated with alpha-blockers were observed in comparison with patients treated with a 5-ARI (adjusted HR: 1.67, 95\% CI: 1.19-2.35; and adjusted HR: $1.51,95 \%$ CI: 1.16-1.97, respectively).

The difference in favour of finasteride remained when excluding patients $(n=161)$ having BPHrelated surgery within four weeks of the start of the BPH treatment initiation (adjusted HR: 1.57, 95\% CI: 1.27-1.94) for alpha-blockers in comparison with 5-ARI. When investigating the risk of surgery related to individual alpha-blockers, there was an increased risk of BPH-related surgeries in comparison with finasteride for alfuzosin, tamsulosin and terazosin (adjusted HR: 1.48, 95\% CI: 1.19-1.83; adjusted HR: $1.60,95 \%$ CI: 1.25-2.06; and adjusted HR: 1.65, 95\% CI: 1.22-2.24, respectively). To take into account the difference between alpha-blockers and finasteride with respect to the time to symptom relief, an additional analysis was

Table 3

Survival from first BPH drug treatment to BPH-related surgery, $<365$ days and $\geq 365$ days

\begin{tabular}{lcccc}
\hline BPH drug use & Number of patients & Number of events $(\%)$ & Crude HR (95\% CI) & Adjusted HR (95\% CI) \\
\hline Duration $<365$ days & & & & 1.00 (reference) \\
$\quad$ 5-ARI & 490 & $88(18.0)$ & $1.03(0.83-1.29)$ & 1.00 (reference) \\
Alpha-blockers & 3497 & $656(18.8)$ & & $1.17(0.93-1.49)$ \\
Duration $\geq 365$ days & & & 1.00 (reference) & 1.00 (reference) \\
5-ARI & 341 & $22(6.5)$ & $1.25(0.78-1.99)$ & $1.53(0.95-2.47)$ \\
Alpha-blockers & 1343 & $94(7.0)$ & \\
\hline
\end{tabular}

HR, hazard ratio; CI, confidence interval; 5-ARI, 5-alpha-reductase inhibitor.

${ }^{a}$ Adjusted hazard ratio for age, calendar year, type of prescriber and chronic disease score. 
conducted where patients who were dispensed finasteride for a period less than 60 days or 180 days, respectively, were excluded. Among these patients, an increased risk of BPH-related surgery was observed for patients treated with alpha-blockers compared with patients treated with 5-ARI (for 60 days: adjusted HR: 2.13, 95\% CI: $1.67-2.73$, for 180 days: adjusted HR: $2.96,95 \%$ CI: $2.13-4.12$, respectively).

\section{Discussion}

In this study, we found that the risk of progressing to BPH-related prostatic surgery was significantly higher for patients treated with alpha-blockers compared with patients treated with 5-ARI. Similar results were obtained by Boyle et al. who performed a retrospective analysis of patient data from the General Practice Research Database between 1996 and 1999. Patients who were treated with the 5-ARI finasteride had a significantly lower risk of experiencing serious complications associated with the progression of $\mathrm{BPH}$ (catheterisation, AUR, surgery) compared to patients using alpha-blockers (RR: 0.50, 95\% CI: 0.35-0.73) [15]. Clifford et al. found a significant lengthening of the period between first diagnosis of LUTS/BPH and surgery between 1992 and 1998, but also found that the interval between treatment initiation and treatment failure, defined as discontinuation or switching of medical therapy or BPH-related prostatic surgery, was significantly shorter for patients receiving the alpha-blockers indoramin and prazosin relative to patients receiving finasteride. However, for more selective alpha-blockers, such as alfuzosin and tamsulosin, no difference relative to patients using finasteride was found using this definition for treatment failure [16].

The reduction in risk of BPH-related surgeries by 5ARIs compared with alpha-blockers may be explained by their mechanism of action. As prostate volume was shown to be an important risk factor for progression of the disease and, consequently, BPH-related surgery [17], reduction of the prostate by 5-ARI-induced inhibition of the conversion of dihydotestosteron to testosterone may have significantly decreased the risk of $\mathrm{BPH}-$-related surgery in comparison to alpha-blockers.

Prospective studies on the natural progression of BPH in population-based studies have become available recently. In a four-year prospective study of men with $\mathrm{BPH}, 10 \%, 24 \%$, and $39 \%$ of patients with mild, moderate or severe baseline symptoms had BPH-prostatic surgery, respectively [18]. In the Olmsted County Study including 2115 community-dwelling men with $\mathrm{BPH}$, it was found that the risk of developing AUR increased three-fold in men with moderate to severe symptoms as compared to men with mild or no symptoms [19].

The general consensus is that $\mathrm{BPH}$ is a progressive disease [20], although the clinical course is difficult to predict for individual patients, as one patient will progress fast, others slowly, while in others symptoms disappear spontaneously $[18,21]$. The most important risk factors for progression of $\mathrm{BPH}$ reported in the medical literature are age, prostate specific antigen (PSA) value and prostatic volume [22]. In a large population-based study on risk factors for $\mathrm{BPH}$, a lack of physical activity, a history of diabetes, and a history of cardiovascular disease were associated with the development of BPH [23]. It is unknown to what extent comorbidity influences progression of BPH symptoms. Hammarsten et al. found that patients with fast-growing BPH had a high prevalence of type 2 diabetes mellitus, hypertension, obesity, and low HDL-cholesterol levels suggesting that these are risk factors for the development of BPH [24]. In our study, adjusting for concomitant diseases by calculating the chronic disease score did not yield different results.

Some limitations of this study need to be addressed. A diagnosis of BPH relies on surrogate measures for $\mathrm{BPH}$, including clinical (symptom scores), physiological (urinary flow rates), anatomical (prostate volume), and biochemical (PSA values) measurements. Although all of these measures capture come component of BPH, none of them are specific for BPH. Surrogate measures are likely to represent a continuum of disease severity without the existence of a threshold. Therefore, an operational definition of $\mathrm{BPH}$ as a dichotomous condition may not be appropriate $[25,26]$. In this study, the decision to start medical therapy for BPH by the consulting physician was based on one or more of these measures. However, it is unknown to what extent practice variation in the treatment of BPH influenced decisions to start therapy or elect for surgical intervention, and to what extent patients who were prescribed alpha-blockers or finasteride differed with respect to baseline characteristics.

Misclassification may have occurred in this study, as no indication for the use of alpha-blockers was available for this study. The alpha-blockers included in this study were specifically indicated for the treatment of $\mathrm{BPH}$. However, patients who were treated for another urological condition that warranted the use of alpha-blockers, such as urinary tract infections or prostatitis, could have been selected. However, when restricting the analysis to patients who filled at least two prescriptions for an alpha-blocker, similar results were obtained.

Another explanation for the observed difference between both drug types might be that patients were 
on a waiting list for prostatic surgery and were given alpha-blockers as relief during the period before the surgery date. Such a bias would overestimate the hazard of prostatic surgery for patients using alphablockers relative to patients using 5-ARIs, as the latter drug would not have been prescribed to this group of patients. However, the exclusion of patients who underwent prostatic surgery within one month from the start of treatment resulted in a similar increased risk of surgery among the users of alpha-blockers. Further, a prognostic bias may have been introduced in this study due to the slower onset of symptom relief associated with 5-ARIs. However, with stratification according to the time between the initiation of drug therapy and time of surgery, similar hazard ratios were observed.

In conclusion, this study has found a difference in the risk of prostatic surgery among patients with BPH who were treated with alpha-blockers compared with patients being treated with 5-ARIs. Both alpha-blockers and 5-ARIs have proven efficacy in the treatment of symptomatic BPH. Additional research on the longterm outcomes and risk factors for the natural progression of BPH is necessary to identify the optimal medical treatment for BPH patients according to their baseline characteristics.

\section{Acknowledgements}

This study was financially supported by an unconditional grant from GlaxoSmithKline, Zeist, The Netherlands. We gratefully thank Dr. M.M. van Riemsdijk for her contribution to the manuscript.

\section{Appendix A}

See Tables A.1 and A.2.

\section{References}

[1] Berry SJ, Coffey DS, Walsh PC, Ewing LL. The development of human benign prostatic hyperplasia with age. J Urol 1984;132(3): 474-9.

[2] Abrams P, Schulman CC, Vaage S. Tamsulosin, a selective alpha $1 \mathrm{c}$-adrenoceptor antagonist: a randomized, controlled trial in patients with benign prostatic 'obstruction' (symptomatic BPH). The European Tamsulosin Study Group. Br J Urol 1995;76(3): $325-36$

[3] Djavan B, Marberger M. A meta-analysis on the efficacy and tolerability of alpha1-adrenoceptor antagonists in patients with lower urinary tract symptoms suggestive of benign prostatic obstruction. Eur Urol 1999;36(1):1-13.
Table A.1

ICD-9-CM codes for BPH and related problems

\begin{tabular}{ll}
\hline ICD-9-CM code & Description \\
\hline 600 & Benign prostatic hyperplasia \\
601 & Prostatitis \\
788 & Symptoms involving urinary system \\
788.9 & Symptoms involving urinary systems, other \\
599 & Disorders of urethra and urinary tract \\
599.6 & Unspecified urinary obstruction \\
788.2 & Acute urinary retention \\
V53.6 & Fitting of urinary device \\
V53.9 & Fitting and adjustment of other device \\
591 & Hydronephrosis \\
592 & Pyelonephrosis \\
594.1 & Bladder calculi \\
599 & Urinary tract infection \\
& anything with a prefix of 594 = stones in \\
& bladder/urinary tract \\
& anything with a prefix of 590 (infection of kidney) \\
& or 593 (disorder of urether + kidney) or 596 \\
(any bladder disorder)
\end{tabular}

Table A.2

$\mathrm{BPH}$ procedure-codes

\begin{tabular}{ll}
\hline Procedure-code & Procedure \\
\hline $5-600$ & Incision of prostate \\
$5-601$ & TURP \\
$5-601.0$ & Electro-resection \\
$5-601.1$ & Cryo \\
$5-601.2$ & Laser \\
$5-601.3$ & RF, MRF radiation \\
$5-601.8$ & Other techniques \\
$5-601.9$ & Non specified \\
$5-602$ & Suprapubic extirpation/destruction of prostate \\
& (including Hryntsak, Freyer) \\
$5-603$ & Retropubic extirpation/destruction of prostate \\
& (including Millin) \\
$5-604$ & Total and radical prostatectomy \\
$5-604.0$ & Total, abdominal, without lymph toilet \\
$5-604.1$ & Total, perineal, without lymph toilet \\
$5-604.2$ & Radical, abdominal with lymph toilet \\
$5-604.3$ & Radical, perineal with lymph toilet \\
$5-604.9$ & Unspecified prostatic surgery \\
$5-605$ & Other extirpations/destruction of prostate \\
\hline
\end{tabular}

[4] Schulman CC, Cortvriend J, Jonas U, Lock TM, Vaage S, Speakman MJ. Tamsulosin: 3-year long-term efficacy and safety in patients with lower urinary tract symptoms suggestive of benign prostatic obstruction: analysis of a European, multinational, multicenter, open-label study. Eur Urol 1999;36(6):609-20.

[5] Lepor H. Long-term efficacy and safety of terazosin in patients with benign prostatic hyperplasia. Terazosin Research Group. Urology 1995;45(3):406-13.

[6] Hudson PB, Boake R, Trachtenberg J, Romas NA, Rosenblatt S, Narayan P, et al. Efficacy of finasteride is maintained in patients with benign prostatic hyperplasia treated for 5 years. The North American Finasteride Study Group. Urology 1999;53(4):690-5. 
[7] Lukacs B, Grange JC, McCarthy C, Comet D. Clinical uroselectivity: a 3-year follow-up in general practice. BPH Group in General Practice. Eur Urol 1998;33(Suppl 2):28-33.

[8] The MTOPS Research Group. The impact of medical therapy on the clinical progression of BPH: results from the MTOPS trial. AUA Annual Meeting, Orlando, FL, 2002 (Abstract 1042), http://www.niddk.nih.gov/welcome/releases/05-28-02abstract.pdf.

[9] Carson JL, Strom BL, Maislin G. Screening for unknown effects of newly marketed drugs. In: Strom BL, editor. Pharmacoepidemiology. 2nd ed. New York: Wiley; 1994. p. 431-47.

[10] Leufkens HG, Urquhart J. Variability in patterns of drug usage. J Pharm Pharmacol 1994;46(Suppl 1):433-7.

[11] Herings RM, de Boer A, Stricker BH, Leufkens HG, Porsius A. Hypoglycaemia associated with use of inhibitors of angiotensin converting enzyme. Lancet 1995;345(8959):1195-8.

[12] Herings RM, Stricker BH, de Boer A, Bakker A, Sturmans F. Benzodiazepines and the risk of falling leading to femur fractures. Dosage more important than elimination half-life. Arch Intern Med 1995;155(16):1801-7.

[13] Von Korff M, Wagner EH, Saunders K. A chronic disease score from automated pharmacy data. J Clin Epidemiol 1992;45(2): 197-203.

[14] Geller J, Kirchenbaum A, Lepor H, Levine AC. Therapeutic controversies: clinical treatment of benign prostatic hyperplasia. J Clin Endocrinol Metab 1995;80(3):745-7.

[15] Boyle P, Roehrborn C, Harkaway R, de la Rosette J, Emberton M, Logie J. 5-alpha reductase inhibitors (5ARIs) provide superior benefits to alpha-blockers in preventing acute urinary retention and surgery. BJU Int 2002;90(Suppl S2):18.

[16] Clifford GM, Logie J, Farmer RD. How do symptoms indicative of $\mathrm{BPH}$ progress in real life practice? The UK experience. Eur Urol 2000;38(Suppl S1):48-53.
[17] Marberger MJ, Andersen JT, Nickel JC, Malice MP, Gabriel M, Pappas $\mathrm{F}$, et al. Prostate volume and serum prostate-specific antigen as predictors of acute urinary retention. Combined experience from three large multinational placebo-controlled trials. Eur Urol 2000;38(5): 563-8.

[18] Barry MJ, Fowler Jr FJ, Bin L, Pitts III JC, Harris CJ, Mulley Jr AG. The natural history of patients with benign prostatic hyperplasia as diagnosed by North American urologists. J Urol 1997;157(1):10-4.

[19] Jacobsen SJ, Jacobson DJ, Girman CJ, Roberts RO, Rhodes T, Guess HA, et al. Natural history of prostatism: risk factors for acute urinary retention. J Urol 1997;158(2):481-7.

[20] Anderson JB, Roehrborn CG, Schalken JA, Emberton M. The progression of benign prostatic hyperplasia: examining the evidence and determining the risk. Eur Urol 2001;39(4):390-9.

[21] Witjes WP, de Wildt MJ, Rosier PF, Caris CT, Debruyne FM, de la Rosette JJ. Variability of clinical and pressure-flow study variables after 6 months of watchful waiting in patients with lower urinary tract symptoms and benign prostatic enlargement. J Urol 1996;156(3): 1026-34.

[22] Djavan B, Nickel JC, de la Rosette J, Abrams P. The urologist view of $\mathrm{BPH}$ progression: Results of an international survey. Eur Urol 2002;41(5):490-6.

[23] Meigs JB, Mohr B, Barry MJ, Collins MM, McKinlay JB. Risk factors for clinical benign prostatic hyperplasia in a community-based population of healthy aging men. J Clin Epidemiol 2001;54(9):935-44.

[24] Hammarsten J, Hogstedt B. Clinical, anthropometric, metabolic and insulin profile of men with fast annual growth rates of benign prostatic hyperplasia. Blood Press 1999;8(1):29-36.

[25] Jacobsen SJ, Girman CJ, Lieber MM. Natural history of benign prostatic hyperplasia. Urology 2001;58(6 Suppl 1):5-16.

[26] Puppo P. Long-term effects on BPH of medical and instrumental therapies. Eur Urol 2001;39(Suppl 6):2-6. 